\title{
Millimeter-Wave Diffraction by a Photo-Induced Plasma Grating
}

\author{
V. A. Manasson, L. S. Sadovnik, A. Moussessian, and D. B. Rutledge, Fellow, IEEE
}

\begin{abstract}
Optical gratings are used extensively for beamsteering in the visible and IR range of the spectrum. Change in the dielectric permittivity of a semiconductor medium resulting from the excitation of a nonequilibrium electron-hole plasma makes it possible to extend this technique to MMW frequencies. A photo-induced plasma grating (PIPG) can be easily rewritten by changing the illumination pattern, so this technique can be used in an optically controllable MMW antennas. Initial experimental work studied the diffraction of MMW propagating along a dielectric waveguide containing a PIPG [1]-[4]. This paper reports on the diffraction of MMW propagating in free space, steered by the PIPG.
\end{abstract}

\section{SEMICONDUCTOR MATERIAL}

A HIGH RESISTIVITY $\left(\rho>10^{3} \mathrm{ohm} \cdot \mathrm{cm}\right)$ silicon ingot was grown by the float-zone technique. Its long carrier lifetime $\left(\tau>10^{-3} \mathrm{~s}\right)$ enabled it to obtain a high electron-hole plasma density at moderate illumination levels. A $75-\mathrm{mm}-$ diameter, 1.9-mm-thick slab was cut from the ingot. To decrease surface recombination, both of the flat sides of the slab were finished by chemical-mechanical polishing.

\section{EXPERIMENTAL SETUP}

To produce a nonequilibrium plasma, we illuminated the slab with a pulsed xenon lamp through a grating mask (Fig. 1), fabricated by printing opaque strips on transparent film, and entirely transparent to MMW. The incident MMW beam was formed by a horn antenna. The combination of the MMW frequency, $92 \mathrm{GHz}$, the refractive index of silicon, 3.45, and the slab thickness, $1.9 \mathrm{~mm}$, satisfied the conditions for suppressing Fresnel reflection at normal incidence. The MMW beam that passed through the silicon slab was detected by a GaAs Schottky diode coupled with a second horn antenna. The detector was mounted on a rotating arm to measure the angular distribution of the diffracted beam. The pumping light pulses were monitored using a reference photodiode that detected pumping light reflected from the slab. Both the rectified signal

Manuscript received January 13, 1995; revised March 20, 1995. This work was supported in part by a contract from ONR (Project Monitor, W. Miceli).

V. A. Manasson and L. S. Sadovnik are with Physical Optics Corporation, Torrance, CA 90501 USA.

A. Moussessian and D. B. Rutledge are with the California Institute of Technology, Passadena, CA 91125 USA.

IEEE Log Number 9413700.

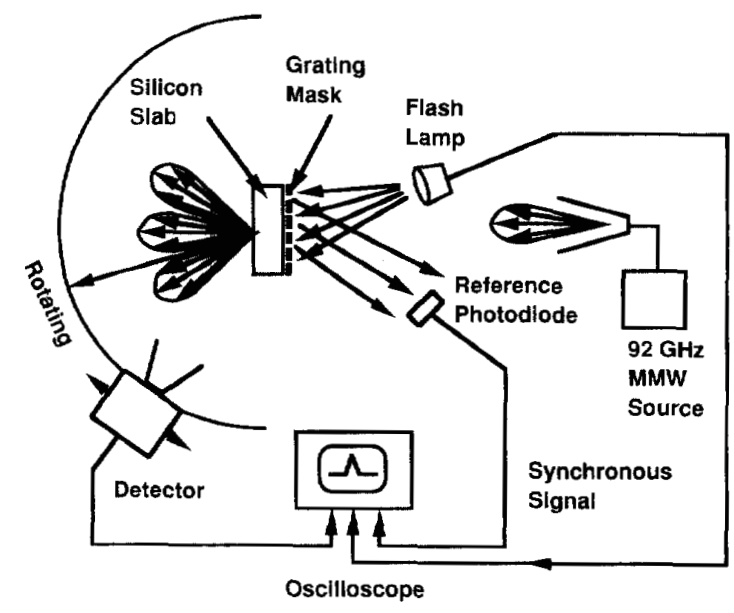

Fig. 1. Experimental setup.

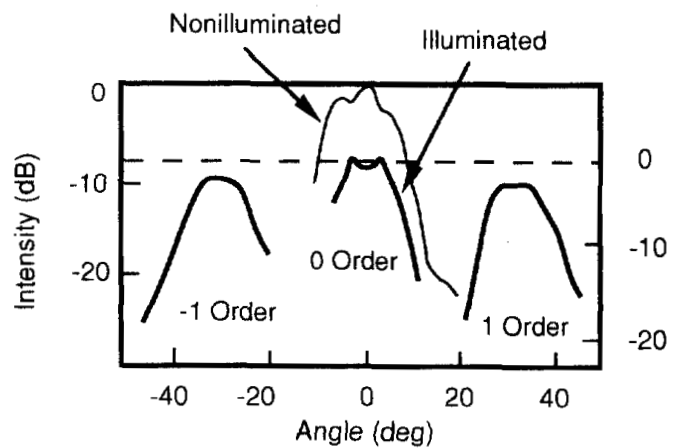

Fig. 2. Beam pattern resulting from diffraction by the PIPG with a $6 \mathrm{~mm}$ period.

from the Schottky diode and the reference signal from the photodiode were displayed on an oscilloscope.

\section{EXPERIMENTAL RESULTS}

Fig. 2 shows the diffraction pattern of the MMW that passed through a silicon slab with a 6-mm-period PIPG. The left scale zero point corresponds to the maximum intensity of the incident MMW beam, and the right scale zero point corresponds to that of the zero-order diffraction beam. The 


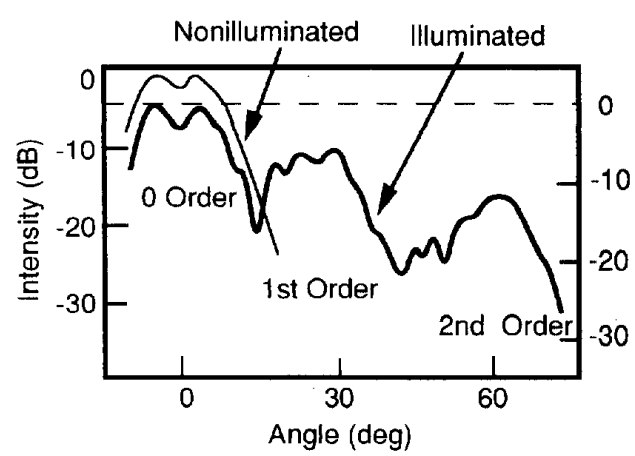

Fig. 3. Beam pattern resulting from diffraction by the PIPG with a $7 \mathrm{~mm}$ period.

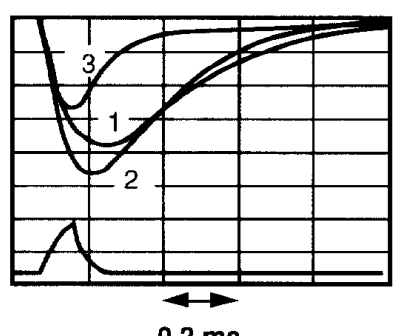

Fig. 4. Time diagrams for the diffracted beam (upper curves) and pumping light (lower curve). Fill factor $=15,23$, and $50 \%$ for curves 1,2 , and 3 . respectively.

intensity of the first order diffraction beam is as high as -9 $\mathrm{dB}$ with respect to the incident beam.

According to classical theory [5], the $m$-order diffraction angle is

$$
\varphi_{m}=\sin ^{-1}(m \lambda / a),
$$

where $\lambda$ is the wavelength of the MMW and $a$ is the grating period. For $\lambda=3.25 \mathrm{~mm}$ and $a=6 \mathrm{~mm}$, the diffraction angles $\varphi_{ \pm 1}= \pm 33^{\circ}$, which is in a good agreement with the results shown in Fig. 2.

Fig. 3 shows one half of the symmetrical pattern obtained using a PIPG with a $7 \mathrm{~mm}$ period. The diffraction maxima correspond to the angles $\varphi_{1}=28^{\circ}$ and $\varphi_{2}=69^{\circ}$, calculated according to (1).

Fig. 4 represents the time diagrams of the first-order diffraction beam (upper curves) and reference signals from the photodiode (lower curves). All of the diffraction curves were obtained with the same grating period $a=6 \mathrm{~mm}$. However, the fill factors ( $\mathrm{FF}=b / a$, where $b$ is the illuminated strip width) of the gratings were different. Curves 1,2 , and 3 correspond to $\mathrm{FF}=15,23$, and $40 \%$, respectively.

The maximum diffraction intensity was reached when the $\mathrm{FF}=23 \%$, far below $50 \%$. A possible explanation for this is the diffusion of photo-induced plasma, which increases the effective strip width opaque to MMW. The diffusion of carriers from illuminated strips to nonilluminated areas can affect their MMW transmission, blocking MMW propagation through the silicon slab after a period of time. The larger the FF, the sooner the diffusion will affect the slab's opacity. This model is in agreement with the behavior of curves 1 through 3 . Curve 3 corresponds to the largest FF and demonstrates the fastest signal degradation after the illuminating pulse has started.

\section{CONCLUSION}

Our experiments show that photo-induced plasma in semiconductors can be used as a convenient way to steer MMW beams. The observed diffraction efficiency is close to the theoretical limit obtainable with a planar transmission diffraction grating [5]. Carrier diffusion affects the diffraction efficiency and must be the focus of future research.

\section{REFERENCES}

[1] A. Rosen, P. J. Stabile, P. Herczfeld, A. Daryoush, and J. K. Butler, "Optically controlled impatt diodes and subsystems," in Proc. 1989 SBMO Int. Microwave Symp. 1989, vol. II, p. 589

[2] P. S. Stabile, R. E. Marx, G. Evans, A. Rosen, R. Amantea, E J. Denlinger, and J. K. Bulter, "Millimeter-wave surface emitters with optically induced gratings," in Proc. IEEE LEOS '90 Conf., 1990, p. 279.

[3] R. Amantea, A. Rosen, P. J. Stabile, W. M. Janton, D. Gilbert, and L. K. Butler, "Optically steered MM-wave grating surface emitter," in LEOS Conf. on Optical Microwave Interactions, Santa Barbara, CA, 1993.

[4] A. Rosen, P. J. Stabile, R. Amantea, W. M. Janton, D. Gilbert, and J. K. Butler, "Active millimeter-wave antenna array steered by optically induced gratings," in Proc. Workshop on Millimeter-Wave Power Generation and Beam Control, 1993, p. 341.

15] R. J. Collier, C. B. Burckhardt, and L. H. Lin, Optical Holography. New York: Academic, 1971.

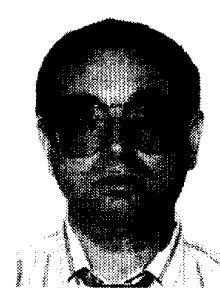

V. A. Manasson received the M.S. degree in electrical engineering from the Moscow Institute of Electronic Manufacturing and the Ph.D. degree in physics from Chernovtsy University (Ukraine).

He is currently a Senior Scientist at Physical Optics Corporation, Torrance, CA. His research in semiconductor physics, microelectronic technology, and optoelectronics has resulted in the design of a number of optoelectronic devices and systems, for example, precision hetero-photodiodes for use in radiometric standards, hetero-photodiodes using a new mechanism of photocurrent amplification, solar cells, polarization sensitive photovoltaic devices, electrooptic and all-optical modulators, discriminators of coherent optical radiation, a new type of hollow optical fiber, and a fiber-optic flow meter. His current research interests include scanning MMW antennas controlled by light. He has to his credit more than 70 publications and conference papers and several patents.

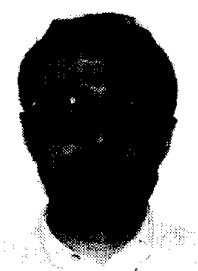

L.S. Sadovnik received the M.S. degree in physical optics from the University of Chernovtsy (Ukraine). $\mathrm{He}$ is completing his Ph.D. at the University of Southern California's Signal and Image Processing Institute.

His expertise is in diffraction optics, holography, and optical image processing. $\mathrm{He}$ is currently a Director of New Technologies at Physical Optics Corporation, Torrance, CA. He completed a theoretical study of high-frequency antireflection gratings, aberration-corrected holographic optical elements, and highly efficient concave gratings. Among his inventions is an innovative algorithm and optical design for a distortion-invariant processor. He proposed and experimentally verified a new principle of phase coding for an all-optical processor. His current research interests include millimeter-wave beamsteering and beamforming controlled by light.. He is the author or co-author of 25 publications and two patents. 


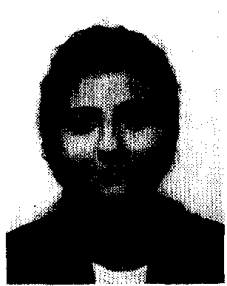

mcirowave circuits.
A. Moussessian was born in Tehran, Iran, on June 21,1966 . She received the B.S. degree in electrical engineering from Iran University of Science and Technology, Tehran, in 1988. In 1992, she received the M.S. degree in electrical engineering from the California Institute of Technology, Pasadena. She is currently a candidate for the Ph.D. degree at Caltech.

Her research interests include microwave and millimeter-wave power combining, beam-steering and computer-aided design and measurement of

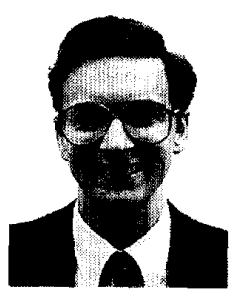

D. B. Rutledge (M'75-S'77-M'77-S'78-M'80SM'89-F'93) received the Ph.D. degree in electrical engineering from the University of California at Berkely in 1980

In 1980 he joined the faculty at the Califorina Institute of Technology, Pasadena, CA, where he is now a Professor of Electrical Engineering. He was a Visiting Scientist at CSIRO, New South Wales, Australia, in the sumer of 1985, and at the Reseach Institute for Electrical Communication Tohoku University, Sendai, Japan, in the spring and summer of 1988 . His research is in developing microwave and millimeterwave integrated circuits and applications, and in software for computer-aided design and measurement. He is co-author with Scot Wedge and Richard Compton fo the software CAd program, Puff, which has over 10000 users worldwide.

Dr. Rutledge is a winner of the NSF Presidential Young Investigator Award and the Japan Society for the Promotion of Science Fellowship. He has been a Distinguished Lecturer for the Antennas and Propagation Society, and is a winner of the 1993 Microwave Prize. 